\title{
Article
}

\section{Primitive Chain Network Simulations of Damping Functions for Shear, Uniaxial, Biaxial and Planar Deformations}

\author{
Kenji FurUichI", Chisato NONOMURA*, Yuichi MASUBUCHI ${ }^{* *}$, Giovanni IANNIRUBERTO ${ }^{* * *}$, \\ Francesco $\mathrm{GRECO}^{* * * *}$, and Giuseppe MARRUCCI ${ }^{* * *}$ \\ ${ }^{*}$ TOYOBO Co., Ltd., Shiga 520-0292, Japan \\ ${ }^{* *}$ Department of Organic and Polymer Materials Chemistry, Tokyo University of Agriculture and Technology, \\ Japan Science and Technology Agency, Tokyo 184-8588, Japan \\ *** Dipartimento di Ingegneria Chimica, Universita' degli studi di Napoli "Federico II" \\ ${ }^{* * * *}$ Istituto per i Materiali Compositi e Biomedici - CNR
}

(Received : October 16, 2006)

\begin{abstract}
Damping functions of entangled polymers for shear, uniaxial, biaxial, and planar deformations, as well as normal stress ratios for shear deformations, were obtained from Brownian simulations making use of the primitive chain network model. To investigate the effect of the force balance over the entanglements and of the convective constraint release mechanism, comparisons with predictions of earlier theories and with experimental data in the literature were performed. It was found that the obtained damping functions are close to the three-chain theory [Marrucci, Greco, and Ianniruberto, Macromol Symp, 158, 57 (2000)] suggesting that the force balance is a dominant correction over the basic Doi-Edwards theory as compared with the effect of convective constraint release. Furthermore, the predicted normal stress ratio in shear, i.e., a quantity very sensitive to the different assumptions, is in good agreement with experiments, suggesting that the combination of force balance, convective constraint release, and other relaxation modes, as accounted for through the primitive chain network model, is quite acceptable.
\end{abstract}

Key Words: Primitive chain network / Entangled polymers / Damping functions / Normal stress ratio / Force balance / Convective constraint release

\section{INTRODUCTION}

Detailed relaxation mechanisms of entangled polymers are still under discussion, though it has been established that relaxation can be described through molecularly-based mechanisms such as reptation ${ }^{1}$, contour-length fluctuations ${ }^{1}$, thermal $^{2)}$ and convective ${ }^{3)}$ constraint release.

In convective constraint release (CCR), as originally proposed by Marrucci ${ }^{3}$, loss of entanglements from a given chain, induced by the convective motion due to the deformation or flow of surrounding chains, generates an accerelation of the relaxation. The CCR has been implemented in constitutive models such as that of Mead, Larson and Doi (MLD) ${ }^{4}$, where the authors show that some basic features in the non-linear rheology of linear polymers are captured. However, it is also known that CCR causes strong damping in the relaxation modulus, and hence that the damping function is underpredicted by, for example, the MLD model as compared with the original theory of Doi and Edwards.
Moving on to a different aspect, force balance (FB) around entanglements has been suggested as a candidate additional mechanism in polymer dynamics. To account for force balance, Marrucci et al. ${ }^{5,6)}$ introduced a simple three-chain model where all chain strands are permanently aligned to the principal axes of the deformation. In spite of its crudeness, the three-chain model seems to fit the experimental damping functions quantitatively.

Relative contributions of CCR and FB to the nonlinear rheology of polymers have not been clarified as yet. Ianniruberto and Marrucci $^{7)}$ proposed a simple constitutive model where CCR affects the relaxation time, and FB is taken into account by adopting the deformation tensor derived by the three-chain model. Although their model qualitatively predicts the observed nonlinearities, the assumed separate influence of CCR and FB on relaxation time and deformation effects, respectively, remains arbitrary. Needless to say, even the tensor emerging from $\mathrm{FB}$, as based on the three-chain model, is questionable due to the assumed alignment of the strands to the principal axes, and to the implicit disregard of detailed

** To whom correspondence should be addressed. 
relaxation effects.

In this study, a direct test of the combined effects of FB and CCR is performed by using the primitive chain network (PCN) simulations $^{8)}$ for several kinds of step strain deformations. Indeed, in PCN simulations, a sliplink network randomly formed in real 3D space relaxes by automatically including network rearrangements due to thermal processes as well as CCR, plus force balance effects on the network nodes.

\section{MODEL AND SIMULATIONS}

Since the PCN model has already been presented $^{8)}$, only a brief description of the model will be given here, together with some details of the specific role played by FB and CCR. In the PCN model, a polymer chain is represented by consecutive segments, and the chains are interconnected through sliplinks to form a $3 \mathrm{D}$ network. State variables of the system are position of the sliplinks, $\{R\}$, number of monomers in each segment, $\{n\}$, and number of segments per chain, $\{Z\}$. Kinetic equations for $\{R\}$ and $\{n\}$, and an algorithm for the change of $\{Z\}$ describe the dynamics of the system. In the absence of flow, the position vector $\mathbf{R}$ of a sliplink obeys the following Langevin-type kinetic equation

$$
\zeta \dot{\mathbf{R}}=\mathbf{F}^{e}-\nabla \mu+\mathbf{F}^{r}
$$

where $\zeta$ is friction coefficient of the node. On the right-hand side, this equation involves the resultant $\mathbf{F}^{e}$ of the tensile elastic forces in the segments emanating from the sliplink, the chemical potential gradient generated by the density field, and a Brownian random force $\mathbf{F}^{r}$ linked to friction so as to fulfill the fluctuation-dissipation theorem. The elastic force $\mathbf{F}^{e}$ is written in the Gaussian form

$$
\mathbf{F}^{e}=\frac{3 k T}{b^{2}} \sum_{j} \frac{\mathbf{r}_{j}}{n_{j}}
$$

where $\mathbf{r}$ is end-to-end vector of chain segment, containing $n$ monomers of length $b$. The sum in Eq. (2) contains four terms because of the binary contact assumption between entangled chains.

The time evolution of $n$ due to sliding of the chain through the sliplinks obeys the following 1D Langevin equation

$$
\frac{1}{2} \zeta \frac{a}{n_{0}} \dot{n}=\frac{3 k T}{b^{2}}\left(\frac{r_{\text {out }}}{n_{\text {out }}}-\frac{r_{\text {in }}}{n_{\text {in }}}\right)+F_{m}{ }^{r}
$$

where $a$ is the average equilibrium distance between consecutive entanglements, and $n_{0}$ is the corresponding monomer number.
The friction coefficient is taken one half that of the node because two subchains (instead of four) are here involved. The first term on the right hand side of Eq. (3) represents the local tension difference of the two chain segments in and out of the sliplink. The second term $\boldsymbol{F}_{\boldsymbol{m}}{ }^{\boldsymbol{r}}$ is a 1D Brownian random force which also fulfills fluctuation-dissipation theorem.

The algorithm for change of $\{Z\}$ is triggered by the $n$ values at chain ends. When $n$ in the end-segments of the chains becomes lower than a given minimum (as a result of the transport of monomers), the corresponding end sliplink is removed. On the contrary, when $n$ in the end-segment exceeds a given maximum, a new sliplink is generated by hooking a nearby segment. The number window for $n$ employed in this study is:

$$
1<\frac{n}{n_{0}}<1+\frac{1}{2}
$$

The $\{Z$ \} change algorithm allows for diffusion of the chains, and hence for rearrangement of the sliplink network.

In the PCN model, FB is taken into account through the Langevin equations (1) and (3). Notice also that FB in the PCN model applies to segments which are randomly oriented at equilibrium, and remain variously orientated even after deformation or flow, whereas in the three-chain model each segment is aligned to one of three orthogonal directions.

Also CCR is naturally taken into account in the PCN model, by the combined dynamics of $\{R\},\{n\}$ and $\{Z\}$. Indeed, under a step deformation, first $\{R\}$ is changed almost affinely (with deviations from affinity due to the force balance). Next, since the change of $\{R\}$ generates an increase of mean segment length, transport of $\{n\}$ is induced by the unbalanced tension along the chains. More specifically, since the chain ends are always at equilibrium, monomers in the chain ends are "sucked in" to release the increased tension in the chain interior. Such a monomer number decrease at the chain ends causes decrease of $\{Z\}$ (through the above mentioned algorithm for $\{Z\}$ change), the latter effect being constraint release as induced by convection (CCR).

In the simulations to be reported below, stress relaxation after step-strains in shear, uniaxial, biaxial and planar deformations were calculated for a monodisperse linear polymer. The molecular weight of the monodisperse polymer is $\langle Z\rangle=9.4$, where $\langle Z\rangle$ is the average number of segments per chain after equilibration, slightly differing from the imposed initial condition of $Z=10$ for all chains due to the algorithm for $\{Z\}$ change. Indeed, even for monodisperse system, equilibration under the assumed dynamics generates an intrinsic distribution of the number of segments per chain. 
The segment number density was fixed at 10 . Periodic boundary conditions were used, with a simulation box of size $16^{3}$. The Lees-Edwards boundary condition was employed for shear deformations. Before applying the deformation, the system was equilibrated by running for 100 time units, a lapse sufficient to relax more than $99 \%$ of any initial stress, as shown later. The calculated results for each strain were taken as ensemble averages over 100 independent simulation runs, with different initial configurations. The linear relaxation modulus $G(t)$ was obtained from the equilibrium selfcorrelation function of the shear stress $\sigma_{s}(t)$.

\section{RESULTS AND DISCUSSION}

\subsection{Relaxation Modulus}

Figure 1 (a) shows the relaxation modulus under step shear deformations, $G(\gamma, t)$. It is confirmed that: i) the linear relaxation modulus $G(t)$ (indicated by a solid line) coincides with $G(\gamma, t)$ at small strains, except for the noise at $t \geq 60$ due to the limited accuracy of the long time statistics; ii) The LodgeMeissner rule ${ }^{9)} N_{1}=\gamma \sigma_{s}(t)$, where $N_{1}$ is the first normal stress difference, is reasonably reproduced (data not shown); iii) time-strain separability holds, as shown in Fig. 1 (b), after a time $t_{k} \approx 30$. (Notice that such a value for $t_{k}$ proves insensitive to the type of deformations, data not shown.) In fact, superposition of the modulus curves by a vertical shift for $t>t_{k}$ defines the damping function $h(\gamma)$ as the required shift factor.

\subsection{Damping Functions}

Figures 2 (a)-(d) show the simulation results for the damping functions which are obtained in shear, uniaxial, biaxial, and planar step deformations, as a function of the respective strains $\gamma, \varepsilon_{U}, \varepsilon_{B}$, and $\varepsilon_{P}$. The predictions of the three-chain model (incorporating FB), of the MLD model (incorporating CCR), of the basic tube theory of Doi and Edwards (DE), and of a recent theory of Greco ${ }^{10)}$ (incorporating the detailed Gaussian statistics in the chain segments) are also shown, together with experimental data for comparison.

The predicted damping functions for all the deformation modes are (from top to bottom in Fig. 2) those from PCN, three-chain model, Greco, DE, and MLD. The MLD predictions for the various deformation modes are taken from the work of Isaki et $a l^{11)}$. Note that MLD shows an overdamping behavior for all deformations, even when compared with $\mathrm{DE}$, which is explained by acceleration of the orientational relaxation of segments induced by CCR. On the other hand, PCN simulations, which involve both FB and CCR, do not show over-damping and, on the contrary, almost coincide with the three-chain model. It so appears that in our code FB somehow suppresses the orientational relaxation due to CCR. Such an effect is indeed conceivable because, in a deformation-induced oriented situation, when a CCR event due to retraction attempts to locally randomize chain orientation, FB somehow brings back the disordered chain, i.e., it forces the disordered segment to realign again to the prevailing orientation. Concerning Greco's theory, it is noted that the (slight) discrepancy with respect to PCN might be due to the different tension law in the entangled chain segments. Indeed, Greco's theory approaches PCN
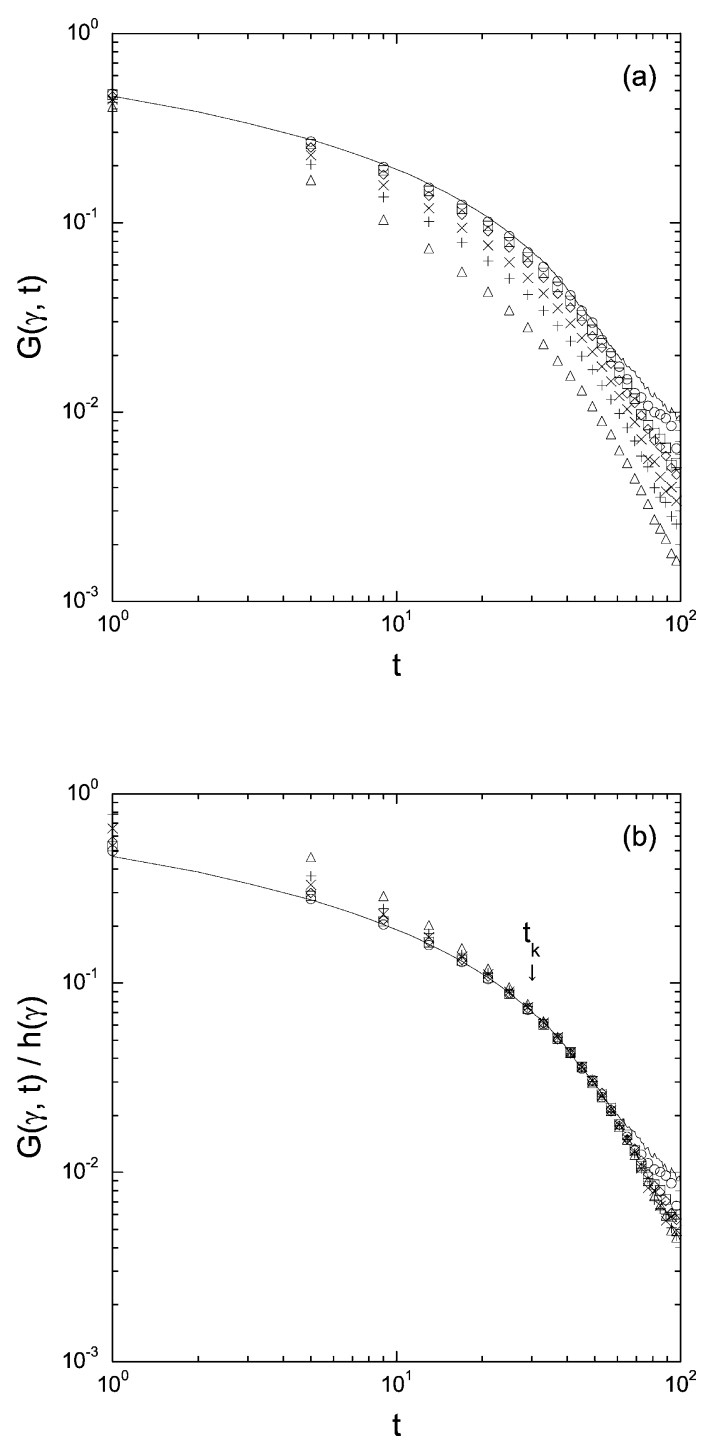

Fig. 1. (a) Shear stress relaxation modulus $G(\gamma, t)$ for various strains. From top to bottom $\gamma=0.5,0.75,1.0,1.5,2.0,3.0$. The solid line is the linear relaxation modulus $G(t)$

(b) Test of time-strain separability for the data in Fig. 1(a). Time $t_{\mathrm{k}}$ indicates the characteristic value below which the time-strain separability is not satisfied. 
results if the average number of Kuhn steps in the segment, $n_{0}$, increases. We used $n_{0}=23$ for Greco's predictions in Fig. 2, since this value has been reported for PS melts. ${ }^{10)}$

Concerning comparison with experimental data ${ }^{11-13)}$, the degree of agreement with predictions varies with the deformation mode. For shear and uniaxial deformations (see Figs. 2(a) and 2(b)) PCN agrees with the experiments in much the same way as the three-chain model, as well as Greco, and slightly better than DE, whereas MLD falls significantly below. It is also noted that, since in the uniaxial deformation mode chain stretch is more significant than in shear, the predictions of PCN might perhaps further improve by replacing the simple linear (Gaussian) springs, as used here, with some finite extensibility law. Even for planar elongation, Fig. 2(d), considering the polydispersity of the sample as well as the technical difficulty to set up the experiment, PCN as well as most of the other theories make a good job, with the exception of MLD. On the other hand, for biaxial deformations, Fig. 2(c), experimental data fall closer to MLD than to the other predictions, PCN and three-chain model included. This would suggest that in biaxial deformations CCR effects somehow dominate on those resulting from FB. Further investigations on this deformation mode from both the experimental and the theoretical viewpoints seem to be required.
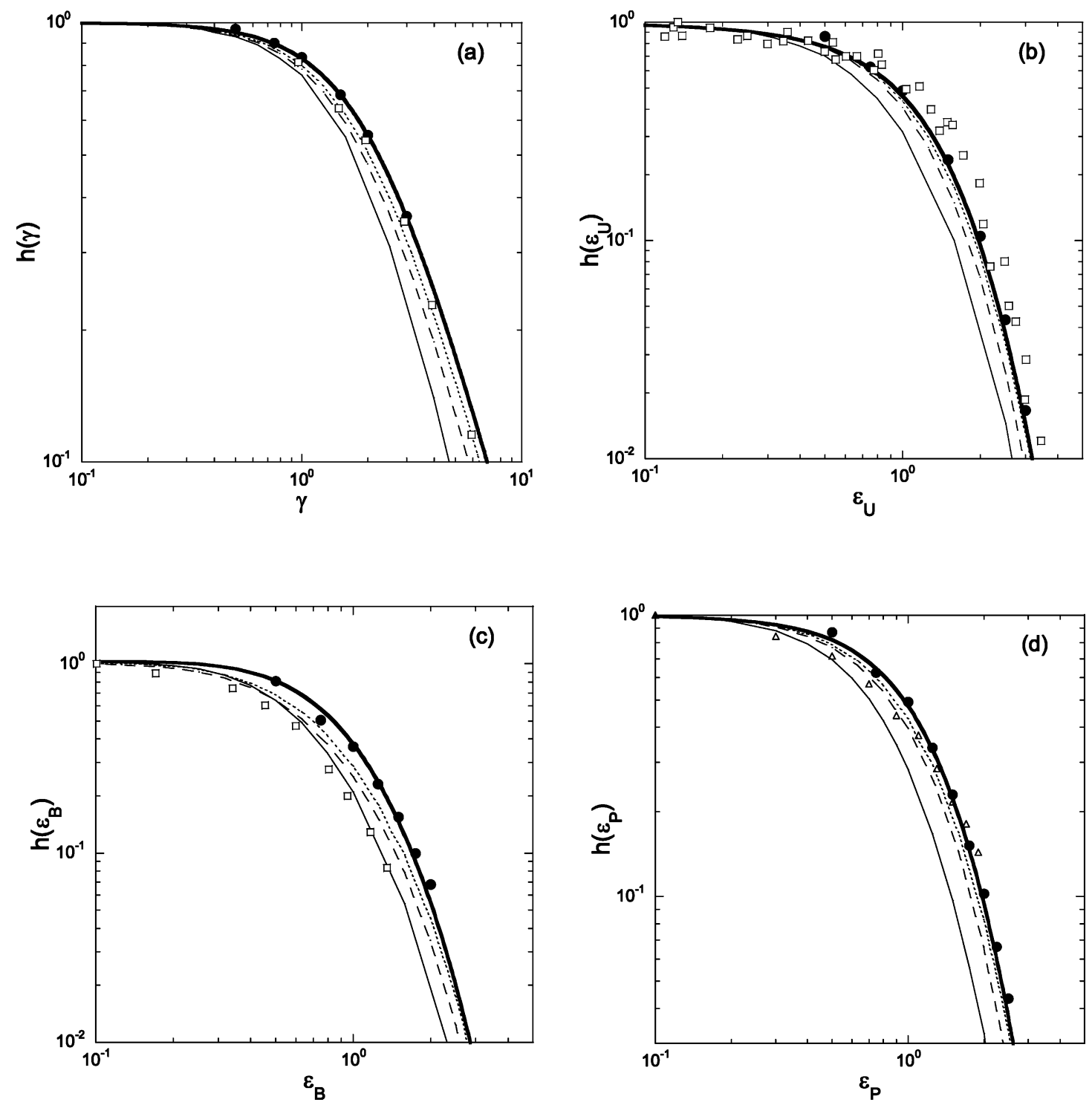

Fig. 2. Damping functions for (a) shear, (b) uniaxial, (c) biaxial, and (d) planar deformations. Closed circles are simulation results, solid lines are three-chain theory ${ }^{5,6}$, dotted lines are Greco theory ${ }^{10)}$, broken lines are Doi-Edwards theory ${ }^{1)}$, and thin solid lines are Mead-Larson-Doi theory ${ }^{4}$. Experimental data represented by open squares in Figs. 2(a)-(c) are from Refs.11) and 12), and open triangles in Fig. 2(d) are data from Ref. 13). 


\subsection{Normal Stress Ratio}

Figure 3 shows the absolute value of the ratio $\Psi(\gamma)$ of secondary to primary normal stress difference in shear deformations, referred to as normal stress ratio. Results from PCN simulations are in between predictions from the threechain model and from the Greco theory, and are consistent with experiments ${ }^{14,15)}$. DE theory gives the lowest $\Psi(\gamma)$ in Fig.3. Notice also that MLD predictions overlap DE in this case, i.e., CCR in the MLD model does not affect $\Psi(\gamma)$. On the other hand, the FB effects, accounted for both in the threechain model and in PCN simulations, generate an increase of $\Psi(\gamma)$, in qualitative agreement with experiments. Here however, at variance with the above reported results on damping functions, there is some discrepancy between PCN and three-chain model. It so appears that the normal stress ratio $\Psi(\gamma)$ (differently from the damping function) is quite sensitive to detailed effects of network rearrangement caused by CCR, and not included in the three-chain model. In other words, the normal stress ratio reveals that the disordering action of CCR is not fully recovered through $\mathrm{FB}$, in better agreement with expectations. Notice finally that the PCN predictions at small strains $(\Psi(\gamma) \sim 0.25)$ are perhaps of limited accuracy, due to large fluctuations in both normal stress differences to be evaluated.

\section{CONCLUSIONS}

Primitive chain network (PCN) simulations were performed for large step deformations, and effects of the convective

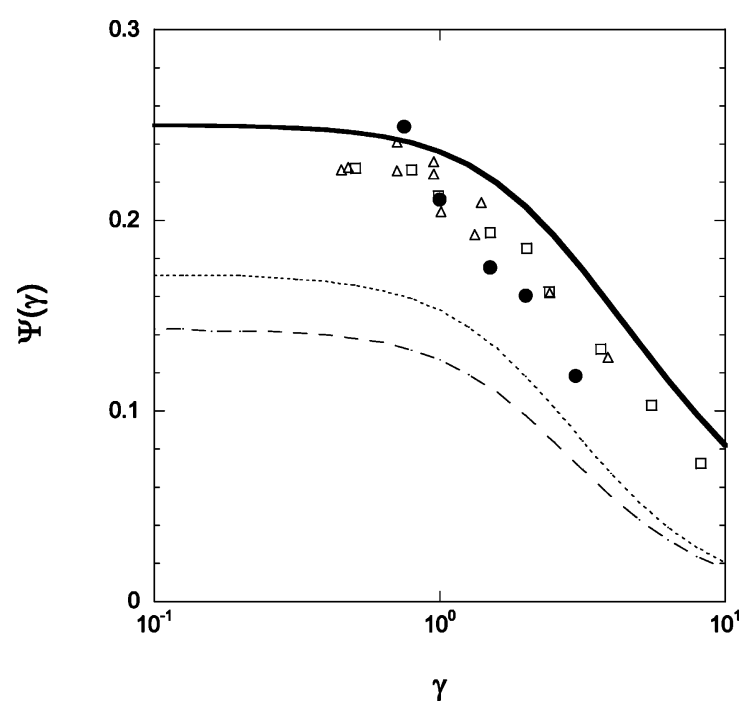

Fig. 3. The normal stress ratio for shear deformations. Closed circles and lines as in Fig. 2, except that DE and MLD curves coincide. Open squares and triangles are data from Brown et al. ${ }^{14)}$ and Olson et al. ${ }^{15)}$, respectively. constraint release (CCR) and of the force balance on entanglements (FB) were investigated. It was found that: i) PCN simulation results for the damping functions are quite close to predictions from the simple three-chain model, thus suggesting that FB may be a needed ingredient in modeling entangled polymers; ii) the damping functions calculated from PCN are fairly close to data available in the literature for shear, uniaxial and planar deformations, whereas PCN predictions for biaxial deformations show some discrepancy, for unknown reasons, from the available data; iii) the PCN normal stress ratio in step shear deformation is in better agreement with experiments than other theories, suggesting that CCR and FB effects are better accounted for in the PCN model.

\section{Acknowledgement}

The authors (KF and $\mathrm{CN}$ ) wish to acknowledge TOYOBO Co., Ltd. for their permission to publish this paper. We thank Dr. T. Isaki and Dr. A. Nishioka for sending us the experimental data of the damping functions. This study was supported in part by Grants in Aid for Scientific Research from the Ministry of Education, Culture, Sports, Science and Technology of Japan.

\section{REFERENCES}

1) Doi M, Edwards SF, "The Theory of Polymer Dynamics", (1986), Oxford University, Oxford.

2) Graessley MM, Adv Polym Sci, 47, 67 (1982).

3) Marrucci G, J Non-Newtonian Fluid Mech, 62, 279 (1996).

4) Mead DW, Larson RG, Doi M, Macromolecules, 31, 7895 (1998).

5) Marrucci G, Greco F, Ianniruberto G, Macromol Symp, 158, 57 (2000).

6) Marrucci G, Greco F, Ianniruberto G, J Rheol, 44, 845 (2000).

7) Ianniruberto G, Marrucci G, J Rheol, 45, 1305 (2001).

8) Masubuchi $Y$, Takimoto J, Koyama K, Ianniruberto G, Greco F, Marrucci G, J Chem Phys, 115, 4387 (2001).

9) Lodge AS, Meissner J, Rheol Acta, 11, 351 (1972).

10) Greco F, Macromolecules, 37, 10079 (2004).

11) Isaki T, Takahashi M, Urakawa O, J Rheol, 47, 1201 (2003).

12) Urakawa $O$, Takahashi $M$, Masuda $T$, Ebrahimi NG, Macromolecules, 28, 7196 (1995).

13) Nishioka A, Takahashi T, Masubuchi Y, Takimoto J, Koyama K, J Non-Newtonian Fluid Mech, 89, 287 (2000).

14) Brown EF, Burghardt WR, Kahvand H, Venerus DC, Rheol Acta, 34, 221 (1995).

15) Olson DJ, Brown EF, Burghardt WR, J Polym Sci Part B. Polym Phys, 36, 2671 (1998). 